\title{
Investigation of the Hyperelastic Material Coated Steel Car Hood Concerning Pedestrian Head Impact Protection using Finite Element Method
}

\author{
M. YAZICI ${ }^{a *}, Y$. CAN $^{b}$ AND H. GÜÇLÜ̈ \\ ${ }^{a}$ Uludağ University Engineering Faculty, Automotive Engineering Department, Bursa, Turkey \\ ${ }^{b}$ R\&D Center, Oyak-Renault, Bursa, Turkey
}

\begin{abstract}
Pedestrians are the high-risk group in vehicle accidents, especially in urban areas. In the world, every year numerous pedestrians die by vehicle impacts. Therefore, the pedestrian protection is one of the most crucial subjects in automotive manufacturers, and many governments are releasing more drastic regulations. However, in the traffic there is a considerable amount of the cars running which have been produced before pedestrian safety regulations. In this study, the pedestrian head impact on an energy absorber material covered steel bonnet was investigated by finite element method. The pedestrian head impact is the first reason for the fatality in the vehiclepedestrian accidents. The pedestrian head-form is used for simulations according to EEVC/WG17 regulations. The foam cover and hood thicknesses are the parameters in the impact simulations. Results of the impact analyses are compared with each other by head injury criterion calculations. The head injury criterion values are accepted for measuring pedestrian's head injury level. In the study, various thickness hyperelastic foams were used in top layer as a cover. Moreover, also developed layered structure designs by steel, and hyperelastic material layers were discussed on light-weighting.
\end{abstract}

DOI: 10.12693/APhysPolA.134.238

PACS/topics: pedestrian protection, finite element method, headform, head injury criterion, hyperelastic materials

\section{Introduction}

Pedestrian injuries continue to be one of the primary public health problems by rising number of the vehicles and peoples in urban areas around the worldwide. Pedestrians comprise $22 \%$ of all road traffic deaths; approximately 275,000 deaths a year globally [1]. Overall, the vehicle components induced more than $85 \%$ of pedestrian injuries, highlighting the importance of the needs in designing pedestrian-friendly vehicles $[2,3]$. Therefore, pedestrian protection is one of the relevant subjects in the modern automotive industry. Numerous improvements in modern vehicles such as the new designs on outer vehicle shape, novel body front-end materials, incorporating the active and passive protection systems into the modern vehicles have been the main research subject in the automotive industry. As a result of this kind of advances, for instance, during the years 2009-2013 in EU countries, the fatality and serious injury rate of the pedestrians reduced by more than $17 \%$ [4].

For serious pedestrian injuries, US data indicates that the head is most frequently injured followed by the lower extremity and torso [5-7]. However, modern vehicle styling reduces the overall risk of pedestrian injuries; numerous old style design vehicle is running in urban traffic [2]. In the presented study, hyperelastic foam cover pads purposed for old-style vehicles to reduce their pedestrian injury and fatality risks. In the presented study, the

*corresponding author; e-mail: myazici@uludag.edu.tr effect of the foam cover thickness and plate thickness on pedestrian protection were investigated by finite element (FE) simulations with child headform impacts. The head injury criterion (HIC) [8] values were calculated to compare pedestrian safety levels.

\section{Materials and method}

\subsection{Problem definition and FE modelling}

The study performed in two stages by using FE simulations with Abaqus ${ }^{\circledR} 14$ software. In the first step, a flat steel plate base covered with hyperelastic foam is analyzed and compared with flat steel plate simulations. The models and boundary conditions are shown in Fig. 1. The second is the FE simulation of a car hood, and hyperelastic foam covered car hood comparatively. The used car hood models in the second stage of the simulation study are presented in Fig. 2. In both steps, a child headform was impacted on the model surfaces. Used child head form model and mesh geometry are given in Fig. 3. The child headform was stroked to the hood top surface and plate with speed of $11.1 \mathrm{~m} / \mathrm{s}$ and 50 degrees from ground plane according to EEVC regulations.

Three different plate thickness of $0.65,2$, and $3 \mathrm{~mm}$ were used to observe plate thickness effect on child headform acceleration. Tie constraints were used in the connection region between parts for FEA model. Encastre boundary conditions were applied to all models.

\subsection{Used materials and materials model}

In FE analysis low carbon steel was used for the car hood structure. The low carbon steel has $7800 \mathrm{~kg} / \mathrm{m}^{3}$ 

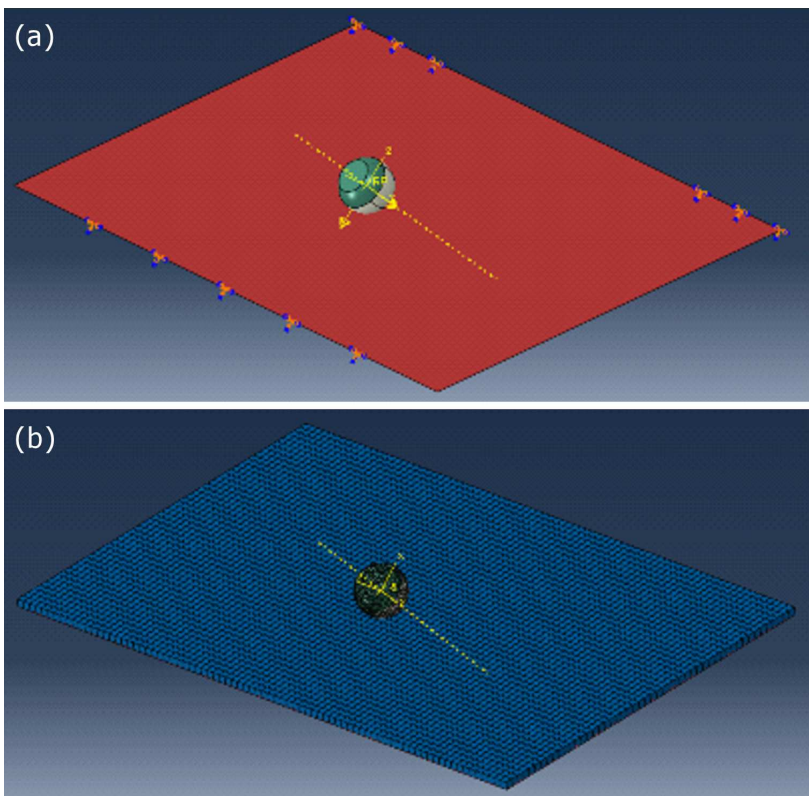

Fig. 1. Steel flat plate - child head impacter simulation model: (a) uncovered steel plate, (b) hyperelastic foam covered steel plate.
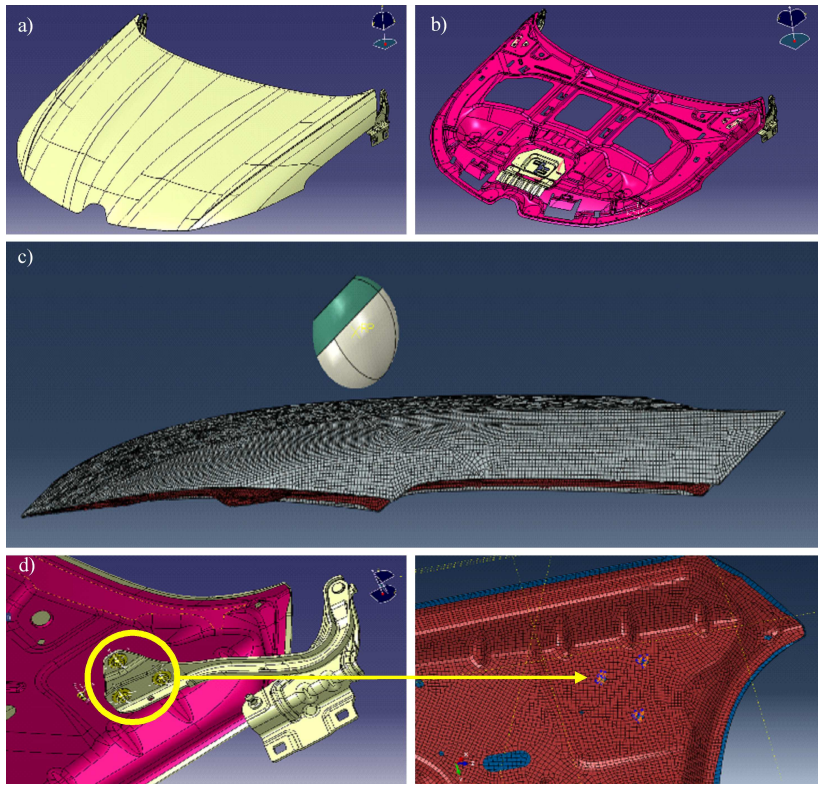

Fig. 2. (a) Outer car hood, (b) inner car hood, (c) FE mesh and child headform, (d) encastre points.

density, 200 GPa Young modulus, 0.3 Poisson ratio, 360 MPa tensile strength and $35 \%$ elongation break physical properties.

The hyperelastic material is described in the FEA software using Ogden hyperelastic material approach. Hyperelastic materials are modeled by strain energy function. In this function, the strain energy density is defined either regarding the principal stretch ratios or the deviatoric strain invariants and volume ratio. To completely characterize the strain energy function, it is the best to
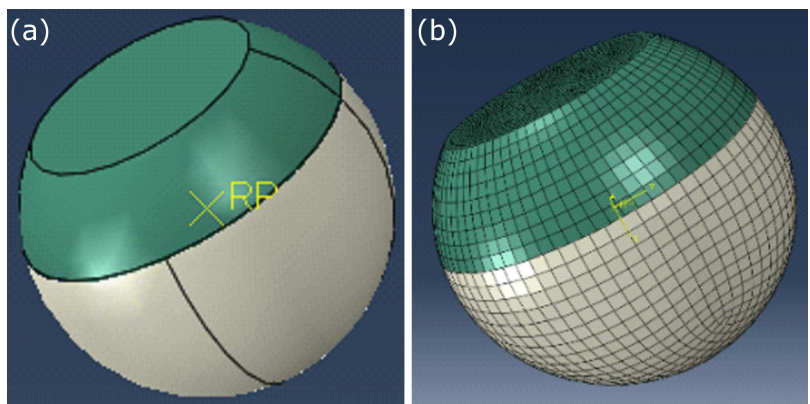

Fig. 3. Child headform impactor FE simulation model: (a) reference point on child headform, (b) FE mesh model of the child headform.

obtain data from several experiments involving different kinds of deformation over the range of strains of interest in the actual application and to use all of these data to determine the parameters [9]. This is particularly true for the phenomenological models, such as the hyperplastic PU foam used in this study where Ogden material parameters were shown in Table I.

TABLE I

Ogden hyperelastic material model parameters [10].

\begin{tabular}{c|c|c}
\hline \hline$N$ & $\mu_{i}$ & $\alpha_{i}$ \\
\hline 1 & 0.0196 & 55.98 \\
2 & 0.0013 & 23.95 \\
3 & 0.0184 & 23.95
\end{tabular}

\section{Results and discussion}

In first simulations, car hood sheet thickness effect obtained by $\mathrm{FE}$ analysis of the three different flat plate thickness. As shown in Fig. 4, the thickest plate-child headform impact results revealed the highest child headform acceleration. Therefore, the thick hood sheet selected to represent the old style cars which are produced before the release of pedestrian protection regulations. To eliminate the shape effects of the hood on pedestrian safety the same geometric models were used.

Figure 5 shows acceleration-time curves by impacting child headform to the $3 \mathrm{~mm}$ steel flat plate covered with 20, 50, and $80 \mathrm{~mm}$ thickness foams. This figure showed that the foam thickness reduced the acceleration time curves' peak values. Moreover, starting points of the curves were shifted from the origin. This means that the child head contact time to the steel surface was delayed. Therefore, the driver of the colliding car earned additional time to reduce vehicle speed. This time delay can provide more survivability to the pedestrians.

In Table II, the summary of the child headform-car hood results was shown. The hood sheet thickness of $0.65 \mathrm{~mm}$ presents today's pedestrian-friendly cars and $1.5 \mathrm{~mm}$ car hood thickness presents old style cars which used to before the pedestrian safety regulations. The results show that a foam cover applied to old style can improve the pedestrian safety level. In the simulations, 


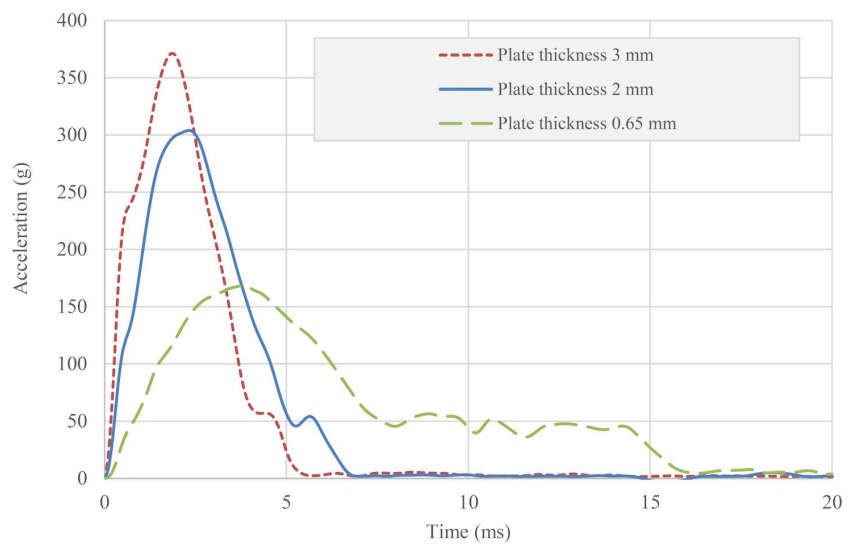

Fig. 4. Inner and outer car hood steel assembly child headform impact acceleration-time curves on hood sheets thicknesses.

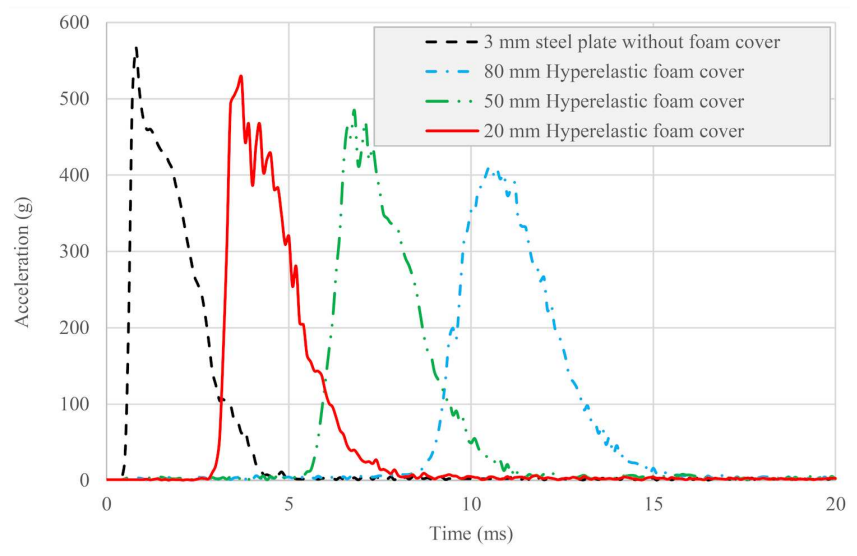

Fig. 5. Child headform impact to $3 \mathrm{~mm}$ steel plate covered with foams as 20,50 , and $80 \mathrm{~mm}$ thickness.

the cover improved the HIC degree in a tiny amount. However, it can be increased by using more crushable or thicker foam covers. Moreover, the child headform maximum stress reduced around $16 \%$ by using foam cover. This means that the foam cover decreased the child skull injury amounts. In the old style cars, the gap between hood and engine components could not be designed as suitable for high hood deflections. However, by foam covering, the deflection results of the hood reduced more than $45 \%$. This improvement gives another occasion to eliminate rigid base effects of under hood substances in the old style cars. The hyperelastic foam cover addition to hood increased the mass of the car hood as the negligible amount $(2.15 \%)$.

\section{Conclusions}

In the presented study, car hood foam cover was proposed for old style cars which have been produced before pedestrian protection regulations and still active in the urban traffic, to improve their pedestrian safety level. The proposed solution was examined by using FE simulations. In the FE simulations, to represent old style car, a high HIC value car hood was modeled by thickening the car hood sheet. The same car hood geometry

\section{TABLE II}

Child headform-hood impact results with and without foam covers. A $-0.65 \mathrm{~mm}$ hood without foam cover, $\mathrm{B}-1.5 \mathrm{~mm}$ hood without foam cover, $\mathrm{C}-1.5$ $\mathrm{mm}$ hood with $20 \mathrm{~mm}$ hyperelastic foam cover.

\begin{tabular}{l|c|c|c}
\hline \hline & A & B & C \\
\hline HIC & 450 & 1563 & 1550 \\
mass $[\mathrm{kg}]$ & 14.29 & 33 & 33.71 \\
displacement [mm] & 73.36 & 22.68 & 12.8 \\
maximum stress & 3.5 & 6.9 & 5.8 \\
on child head cover [MPa] & & &
\end{tabular}

was used to eliminate shape effect in all cases for the pedestrian-friendly modern car (thin hood sheet), unsafe cars for pedestrians (thick hood sheet) and foam cover. Obtained enhancements for the pedestrian safety can be increased by using more crushable and energy absorber foam covers. It was observed that the foam cover could reduce the pedestrian injury level and the foam thickness contributes positively to pedestrian safety. The main reasons are the improved energy absorption capability and gained additional time for driver breaking action. The results also show that, by using a foam cover on the car hood, the magnitude of the reaction forces on the pedestrian head can be reduced. Finally, an application of foam cover on any car hood improved the pedestrian safety level of the car with insignificant increase in the weight of the car hood.

\section{Acknowledgments}

The authors would like to thank Oyak-Renault Automotive Company for their support.

\section{References}

[1] World Health Organization, Global Status Report on Road Safety (2015), Italy.

[2] D. Valladares, J.J. Alba, I. Altubo, Adv. Mech. Eng. 9, 1 (2017).

[3] J. Hu, K.D. Klinich, Int. J. Vehicle Safety 8, 22 (2015).

[4] P. Olszewski, B. Osińska, P. Szagała, P. Skoczyński, A. Zielińska, Arch. Civ. Eng. 62, 149 (2016).

[5] R. Fredriksson, E. Rosén, A. Kullgren, Accid. Anal. Prev. 42, 1672 (2010).

[6] D. Longhitano, B. Henary, K. Bhalla, J. Ivarsson, J. Crandall, SAE Technical Paper, 2005-01-1876 (2005).

[7] G. Zhang, L. Cao, J. Hu, K.H. Yang, Ann. Adv. Automot. Med. 52, 199 (2008).

[8] C. Pinecki, R. Zeitouni, in: The 20th Int. Techn. Conf. on the Enhanced Safety of Vehicles Conference (ESV), Lyon (France), 2007, Paper No. 07-0307-W.

[10] G. Liang, K. Chandrashekhara, Eng. Struct. 30, 2002 (2008).

[11] M.L. Ju, S. Mezghani, H. Jmal, R. Dupuis, E. Aubry, Cell. Polym. 32, 21 (2013). 